\title{
Poils épidermiques, types stomatiques et taxonomie chez les morphotypes de karité Vitellaria paradoxa C.F. Gaertn subsp. paradoxa)
}

\author{
Christophe DJEKOTA ${ }^{1 *}$, Mame Samba MBAYE ${ }^{2 *}$, Doudou DIOP ${ }^{3 *}$, Kandioura NOBA ${ }^{2 *}$ \\ 1 * Faculté des Sciences Exactes et Appliquées. Département de Biologie, Université de N'Djaména, B.P 1027, Tchad, Email: \\ cdjekota@yahoo.fr \\ 2* Faculté des Sciences et Techniques. Département de Biologie Végétale, Laboratoire de Botanique et Biodiversité. Université \\ CheikhAnta DiopDakar,B.P5005 Sénégal,Email:knoba@ucad.sn,.msmbaye@yahoo.fr, \\ 3* Laboratoire de Botanique. IFAN-CAD, Dakar, B.P. 206, Fann, Sénégal, Email: diopdoudon@,hotmail.com
}

Mots-clés : Vitellaria paradoxa paradoxa, poils épidermiques, types stomatiques, taxonomie micro morphologique.

Keywords: Vitellaria paradoxa paradoxa, epidermal hairs, stomatal types, micro morphological taxonomy.

Publication date 31/07/2020, http://m.elewa.org/Journals/about-japs/

1 RESUME

Les poils épidermiques et les types stomatiques chez les morphotypes de karité (Vitellaria paradoxa C.F. Gaertn subsp. paradoxa) ont été étudiés au Tchad en 2010, de Juillet à Septembre. L'objectif est la recherche des caractères micro morphologiques discriminants susceptibles d'améliorer l'identification des morphotypes de cette espèce. En fait, des morphotypes de karité ont été décrits et nommés par les ruraux dans la province du Mandoul au Tchad. Les amandes de karité sont transformées en beurre, ce qui lui confère une importance sur le plan socio-économique. Des fragments de bourgeon apical prélevés sur des jeunes plantes issues de la germination des graines des cinq (5) morphotypes ont permis d'observer les types de poils épidermiques. Aussi, des échantillons de feuilles prélevés distinctement de chaque morphotype ont été préparés selon la méthode de Barfod (1988). Cette méthode a été privilégiée car l'épiderme de la feuille de karité n'est pas facilement détachable. Elle consiste à bouillir les échantillons de feuilles dans de l'eau distillée pendant 10 minutes puis ils sont trempés dans l'acide nitrique à $40 \%$ pendant 16 à 20 heures. Cette opération a permis de ramollir le mésophyle et facilite la desquamation de la cuticule. La surface du fragment de la feuille ramollie placée sur une lame de microscope dans une goutte d'eau est grattée délicatement et progressivement à l'aide du bord de ciseaux jusqu'à ce qu'un fragment d'épiderme transparent apparaisse. Les épidermes foliaires ainsi obtenus sont placés dans du Lugol pendant 10 minutes puis montés après rinçage entre lame et lamelle dans la gélatine glycérinée et observés au microscope optique de type MOTIC. Au total 30 dénombrements ont été effectués sur les deux faces soit en moyenne 6 observations par morphotype. Ces dénombrements ont permis de calculer la densité stomatique et l'indice stomatique de chaque morphotype. La densité stomatique est la moyenne des 6 dénombrements par unité de surface $\left(\mathrm{mm}^{2}\right)$. Les résultats ont montré 4 groupes de morphotypes: 1- des poils épidermiques simples longs observés chez le morphotype A, appelé localement «Bogrombaye»; 2- des poils simples courts observés 
chez les morphotypes C, E et F « Komane, Mbabète, Ngoïtokoro "; 3- des poils glanduleux longs observés chez le morphotype D "Meingré " et; 4- des poils glanduleux courts observés chez le morphotype B "Kiankos». De plus, des stomates de type anomocytique périgène à subsidiaire dicyclique sont observés chez tous les morphotypes étudiés. La densité stomatique évaluée est $362 \pm 5 \mathrm{st} / \mathrm{mm}^{2}$ et l'indice stomatique varie de $45,1 \pm 0,7 \% \mathrm{chez}$ les morphotypes de karité étudiés. Ces connaissances pourraient améliorer la systématique des morphotypes et fournir une base pour la sélection du matériel végétal approprié pour les programmes locaux de reboisement et/ou pour la production agronomique, car le beurre de cette espèce est de plus en plus sollicité.

Epidermal hair, stomatal types and taxonomy in shea morphotypes (Vitellaria paradoxa C.F. Gaertn subsp. Paradoxa)

\section{ABSTRACT}

Epidermal hair and stomatal types in shea morphotypes (Vitellaria paradoxa C.F. Gaertn subsp. paradoxa) were studied in Chad in 2010, from July to September. The objective is the search for discriminating micro morphological characters likely to improve the identification of the morphotypes of this species. In fact, shea morphotypes have been described and named by rural people in the province of Mandoul in Chad. Shea kernels are transformed into butter, which gives it socio-economic importance. Fragments of the apical bud taken from young plants from the germination of the seeds of the five (5) morphotypes made it possible to observe the types of epidermal hair. Also, leaf samples taken separately from each morphotype were prepared according to the method of Barfod (1988). This method was preferred because the epidermis of the shea leaf is not easily detachable. It involves boiling the leaf samples in distilled water for 10 minutes and then soaking them in $40 \%$ nitric acid for 16-20 hours. This operation allowed to soften the mesophyle and facilitates the scaling of the cuticle. The surface of the fragment of the softened leaf placed on a microscope slide in a drop of water is gently and gradually scraped off using the edge of the scissors until a transparent epidermis fragment appears. The leaf epidermis thus obtained are placed in Lugol for 10 minutes and then mounted after rinsing between slide and coverslip in glycerol gelatin and observed under an optical microscope of the MOTIC type. A total of 30 counts were made on both sides, an average of 6 observations per morphotype. These counts made it possible to calculate the stomatal density and the stomatic index of each morphotype. The stomatal density is the average of the 6 counts per unit area $\left(\mathrm{mm}^{2}\right)$. The results showed 4 groups of morphotypes: 1- long single epidermal hairs observed in morphotype A, locally called "Bogrombaye"; 2- short simple hairs observed in morphotypes C, E and F "Komane, Mbabète, Ngoïtokoro"; 3- long glandular hairs observed in the morphotype D "Meingré" and; 4- short glandular hairs observed in morphotype B "Kiankos". In addition, stomata of the perigenic anomocytic type with dicyclic subsidiary are observed in all the morphotypes studied. The stomatal density evaluated is $362 \pm 5 \mathrm{st} / \mathrm{mm}^{2}$ and the stomatic index varies from $45.1 \pm 0.7 \%$ in the shea morphotypes studied. This knowledge could improve the system of morphotypes and provide a basis for the selection of appropriate plant material for local reforestation programs and / or for agronomic production, as butter from this species is in increasing demand. 


\section{INTRODUCTION}

Les peuplements naturels du karité connaissent une forte dégradation sous la pression anthropique, les aléas climatiques et les attaques parasitaires. La demande future en beurre de karité risque d'être compromise si l'on continue à n'exploiter que des arbres sauvages. La dégradation de la ressource se traduit aussi par une perte de la biodiversité (Djekota, 2008; Soro et al., 2004a; 2004b). Le karité est considéré comme un arbre sacré à cause de ses multiples utilisations. Il produit des fruits à pulpe comestible contenant des amandes riches en matière grasse, connue sous le nom de beurre de karité. Celui-ci est utilisé par les populations locales dans l'alimentation, la cosmétique et la pharmacopée (Soro et al., 2004b; Djekota 2014b). Dans son aire de répartition, Vitellaria paradoxa subsp. paradoxa présente une certaine hétérogénéité mentionnée par plusieurs auteurs. Arbonnier, (2000); Byakagaba et al., (2011); Gwali et al., (2012); Lamien et al., (2007); Nafan et al., (2007); Nyarko et al., (2012); Sanou et al., (2006); Ugese et al., (2010) ; Djekota (2014a) ont signalé dans les parcs à karité de leur pays respectif, chez cette sous-espèce la variabilité morphologique et taxonomie d'au moins cinq (5) morphotypes. Ces auteurs ont montré que ces taxa sont reconnaissables par leurs caractères végétatifs notamment la longueur du pétiole, la longueur du limbe, la largeur du limbe mais aussi par leurs caractères reproducteurs (longueur du pédoncule, longueur du fruit, largeur du fruit, longueur de la noix et largeur de la noix). Dans la province du Mandoul au Tchad, les caractéristiques des

\section{MATERIEL ET METHODES}

3.1 Présentation du site d'étude : La Département de Koumra, chef-lieu de la Province du Mandoul est l'une des 7 provinces du Tchad dans lesquelles poussent naturellement le karité sur les 23 que compte le pays (Djekota et al., 2014c). Elle est située dans la zone méridionale du Tchad entre le $9^{\mathrm{e}} / / 35^{\prime}$ et $8^{\mathrm{e}} / / 30^{\prime}$. Elle est soumise à un climat tropical constituants physico chimiques des beurres de ces cinq (5) morphotypes sont connues (Mbaiguinam et al., 2007; Mohagir, 2009). Toutefois, ils ont relevé que la grande variabilité phénotypique des caractères du karité probablement liée à l'action des facteurs écologiques, rend leur utilisation délicate pour la discrimination claire et précise de ces morphotypes. Dans un même parc à karité, il est facile de constater que les fruits de certains morphotypes n'arrivent pas à maturité à la même période. Cette période de maturité agronomique des fruits qui s'étale de juin à octobre semble présenter certains morphotypes comme précoces et d'autres comme des morphotypes tardifs. (Djekota et al., 2014a). Les caractères micro morphologiques et en particulier les caractéristiques des poils épidermiques et les types stomatiques ont été très peu utilisés jusqu'ici dans la systématique du karité. Cependant, Diop, (2008) et Mbaye et al., (2001) ont montré l'importance des caractères micro morphologiques qui complètent pertinemment les observations au plan macro morphologique et permettent de surmonter de nombreuses difficultés dans la distinction des espèces végétales. La présente étude est entreprise pour améliorer les connaissances de ces morphotypes. Il s'agit de rechercher les caractères micro morphologiques discriminants susceptibles d'améliorer l'identification des morphotypes et proposer une clé de détermination des morphotypes basée sur les caractères micro morphologiques étudiés.

de type soudanien caractérisé par l'alternance d'une saison de pluie et d'une saison sèche de durée plus ou moins égale. Les pluies annuelles sont comprises entre 900 et $1200 \mathrm{~mm}$. La plupart des types de sols sont exondés et par endroit inondables. Il ressent un profil sableux en surface généralement peu compacte. Ils sont relativement perméables. La formation végétale 
typique est la savane arborée et boisée (SIDRAT, 2013a). Le village de Kemkian où le matériel végétal a été collecté est situé entre $9^{\circ} 00^{\prime}$ Nord et $17^{\circ} 21^{\prime}$ "Est à l'Ouest de la ville de Koumra. C'est un terroir villageois d'environ 225 ha qui compte 1031 habitants INSEED (2012). Les sols varient d'un type argilo- limoneux, sablonneux ou latéritique. La formation végétale est à dominance savane arbustive. Les activités principales de la population sont: l'agriculture, la pêche, l'apiculture et la chasse. Les caractéristiques du site d'étude sont résumées sur le Tableau 1.

Tableau 1 : Caractéristiques du site d'étude.

\begin{tabular}{l|l|l|l|l|l|l|l|l}
\hline Site & $\begin{array}{l}\text { Latitude } \\
\text { Longitude }\end{array}$ & Localisation & $\begin{array}{l}\text { Surface } \\
\text { terroir }\end{array}$ & $\begin{array}{l}\text { Population } \\
\text { (en 2009) }\end{array}$ & $\begin{array}{l}\text { Type de } \\
\text { sol }\end{array}$ & $\begin{array}{l}\text { Formation } \\
\text { végétale }\end{array}$ & $\begin{array}{l}\text { Activités } \\
\text { principales }\end{array}$ & Pluviométrie \\
\hline Village & $9^{\circ} 00^{\prime} \mathrm{N}$ & $\begin{array}{l}17 \mathrm{~km} \text { à } \\
1^{\circ} \text { 'Ouest de } \\
\text { Kemkian }\end{array}$ & $225 \mathrm{ha}$ & $\begin{array}{l}1031 \\
\text { habitants }\end{array}$ & $\begin{array}{l}\text { Agilo- } \\
\text { limoneux } \\
\text { ou } \\
\text { sablonneux, } \\
\text { latéritique }\end{array}$ & $\begin{array}{l}\text { Savane } \\
\text { arbustive }\end{array}$ & $\begin{array}{l}\text { Agriculture, } \\
\text { pêche, } \\
\text { maraîchage } \\
\text { apiculture } \\
\text { et chasse }\end{array}$ & $\begin{array}{l}1200 \mathrm{~mm} \\
\text { en moyenne }\end{array}$ \\
\hline
\end{tabular}

Les populations rurales qui exploitent les sous produits de karité dans le terroir de Kemkian ont une bonne connaissance ethnobotanique des morphotypes de cette espèce (Mbaïguinam et al., 2007 ; Djekota, 2008). Le parc à karité de
Kemkian a été un des trois (3) sites étudiés par Djekota et al, (2014a), pour la détermination de la variabilité des caractères macromorphologiques des cinq morphotypes.

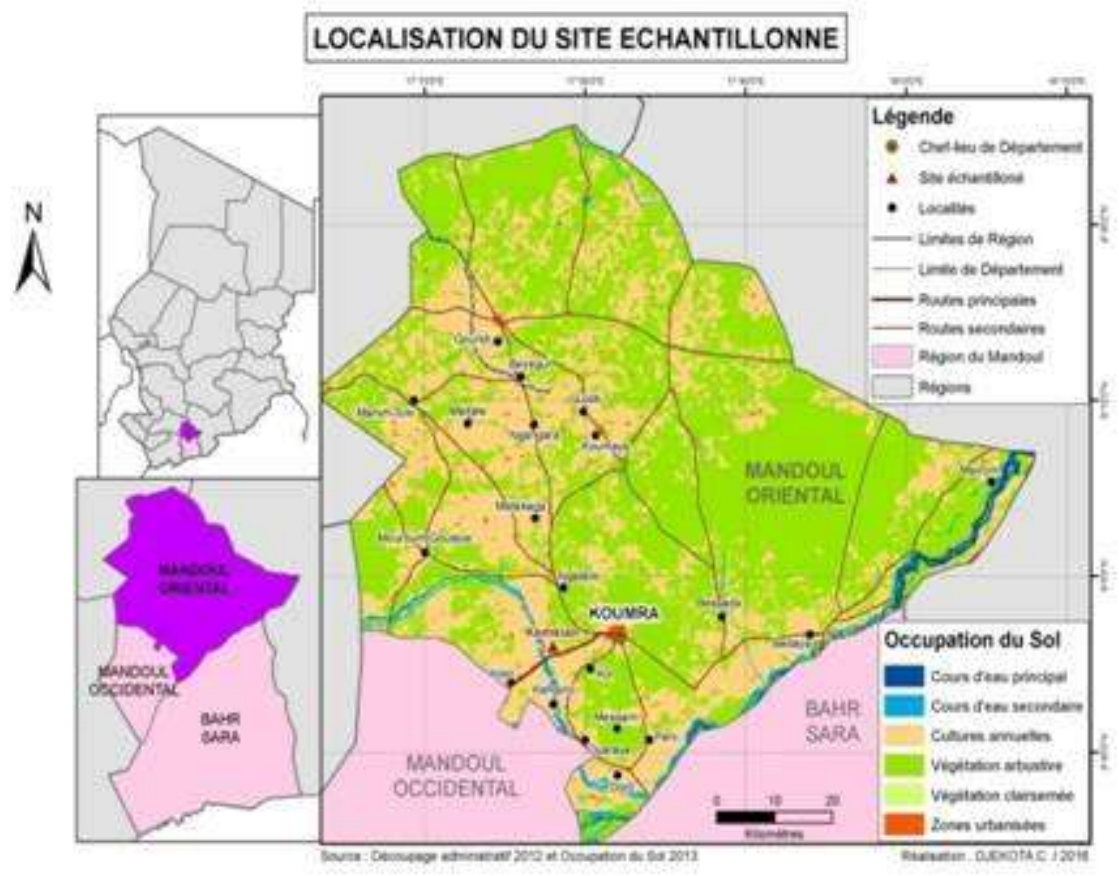

Source: CNRD, Découpage administratif 2012 et Occupation du sol 2013.

Localisation de la zone d'étude : Village de KEMKIAN dans la région du Mandoul oriental au Sud du Tchad. 
3.2 Matériel végétal : Le matériel végétal utilisé pour cette étude est en provenance de Kemkian. Il est constitué d'épidermes des feuilles juvéniles fraîches et de bourgeons apicaux prélevés sur des jeunes plantes au stade 3 à 4 feuilles obtenues de la germination des semences de chaque morphotype de karité.

3.3 Méthodes : Dans le parc à karité de Kemkian, dix (10) graines de fruits mûrs de chaque morphotype ont été récoltées sur les pieds de karité. Ces graines ont été mises en germination en milieu réel dans des pots en plastique de dimensions $20 \times 35 \mathrm{~cm}$ portant des étiquettes d'un code correspondant à chaque morphotype. Après 45 jours de germination, des plantules au stade 3 à 4 feuilles de ces différents morphotypes ont été prélevées pour l'étude des caractères micro morphologiques.

3.3.1. Etude des poils: L'étude de la variabilité des types de poils, leur forme, leur taille et leur structure a été menée en prélevant une portion du bourgeon apical de la plantule de chaque morphotype. L'échantillon est monté dans la gélatine glycérinée entre lame et lamelle et observée au microscope optique de type MOTIC, avec des prises de vues (Planche II, Fig. 1a - 4a).

3.3.2 Etude des stomates : Des échantillons de feuilles prélevés distinctement de chaque morphotype ont été préparés selon la méthode de Barfod (1988). Cette méthode a été privilégiée car l'épiderme de la feuille de karité n'est pas facilement détachable. Elle consiste à bouillir les échantillons de feuilles dans de l'eau distillée pendant 10 minutes puis ils sont trempés dans l'acide nitrique à $40 \%$ pendant 16 à 20 heures. Cette opération a permis de ramollir le mésophyle et facilite la desquamation de la cuticule. La surface du fragment de la feuille ramollie placée sur une lame de microscope dans une goutte d'eau est grattée délicatement et progressivement à l'aide du bord de ciseaux jusqu'à ce qu'un fragment d'épiderme transparent apparaisse. Les épidermes foliaires ainsi prélevés sur les 2 faces des feuilles (supérieure et inférieure) dans des positions identiques sur le limbe sont placés dans du Lugol pendant 10 minutes puis montés après rinçage entre lame et lamelle dans la gélatine glycérinée et observés au microscope optique de type MOTIC, avec des prises de vues (Planche I, Fig. 3 et 4). Les stomates et les cellules épidermiques ont été dénombrés sur des portions de $1 \mathrm{~mm}^{2}$ d'épiderme prélevé dans des régions identiques sur le limbe. Cette technique utilisée par plusieurs biologistes a permis de compter les stomates sans difficulté majeure (Turquet, 1910 ; Guyot, 1966 ; Couderc, 1979; Saadoun \& Decamps, 1991 ; Decamps et al., 1993 ; Noba, 1990 ; Diop, 2008). Les types stomatiques ont été caractérisés en utilisant la classification de Metcalfe \& Chalk (1950) qui est généralement utilisée chez les Dictylédones. Cette classification définit les 4 types suivants :

- type anomocytique ou type Renonculaceae où les stomates sont entourés de plusieurs cellules annexes qui ressemblent aux cellules épidermiques banales (on peut admettre qu'il n'y a pas de cellules annexes typiques chez les Renonculaceae);

- type anisocytique ou type Brassiceae où les stomates sont entourés de 3 cellules annexes distinctes dont l'une est nettement plus petite que les deux autres ;

- type paracytique ou type Rubiaceae où les stomates sont flanqués de cellules annexes disposées parallèlement au grand axe de l'ostiole ;

- type diacytique ou type Caryophyllaceae où les stomates sont entourés d'une paire de cellules annexes dont la paroi commune est perpendiculaire au grand axe de l'ostiole.

3.3.3 Analyse statistique des données : Au total 30 dénombrements ont été effectués sur les deux faces soit en moyenne 6 observations par morphotype. Ces dénombrements ont permis de calculer la densité stomatique (Ds) et l'indice stomatique (Is) chez chaque morphotype. La densité stomatique est la moyenne des 6 dénombrements par unité de surface $\left(\mathrm{mm}^{2}\right)$. Après l'évaluation des moyennes des stomates et des cellules épidermiques banales par faces de feuille par morphotype, le 
calcul d'indice stomatique (Is) représentant la fréquence relative c'est-à-dire le nombre de stomates (al) pour le morphotype $l$ par le nombre total de cellules épidermiques $n=a l+$ $b l ; b l$ représentant les cellules épidermiques banales a été fait :

$$
I s=\frac{a l}{a l+b l} \times 100=I s=\frac{a l}{n l} \times 100
$$

Le test de comparaison des proportions a été effectué sous XLSTAT pour apprécier les

\section{RESULTATS et DISCUSSION}

\subsection{Caractères des poils des bourgeons} apicaux : Les poils observés sur les bourgeons apicaux des cinq (5) morphotypes étudiés ont une structure semblable. Il ressort que les morphotypes du karité sont très proches les unes des autres Ce sont des poils simples pluricellulaires de différentes tailles (Planche II). Toutefois on peut distinguer principalement 3 groupes à partir de la taille de poils observés chez chacun des morphotypes étudiés :

1). le groupe de morphotypes à poils courts de 100 à $150 \mu$ (photos 1 a et $2 \mathrm{a})$;

2). le groupe de morphotypes à poils moyens de 1 à $1,5 \mathrm{~mm}$ (photo $4 \mathrm{a})$; différences des valeurs des indices stomatiques. $\mathrm{Au}$ seuil de signification $\alpha=0.05$ a été déterminé $t$ (à lire dans la table de fonction de répartition de loi normale réduite) :

si $\varepsilon \geq 1,96$ l'hypothèse d'égalité des 2 proportions a été rejetée ;

si $\varepsilon \leq 1,96$ l'hypothèse d'égalité des 2 proportions a été acceptée avec un risque d'erreur de $5 \%$.

3). le groupe de morphotypes à poils long de plus de $2 \mathrm{~m}$ (photo $3 \mathrm{a}$ ).

Des types de poils simples et/ou pluricellulaires de différentes tailles ont été observés par Diop (2008) sur les limbes de la feuille de 23 espèces du genre Ficus L.

Belhadj et al., (2007a) ont montré que les poils de différentes tailles chez une plante lui permet de réduire les pertes d'eau, d'excréter des substances comme la cire, de se protéger des rayons ultraviolets et de maintenir une certaines fraicheurs.

Planche I : Différents types stomatiques rencontrés sur le limbe des cinq (5) morphotypes de l'espèce Vitellaria paradoxa sous-espèce paradoxa.
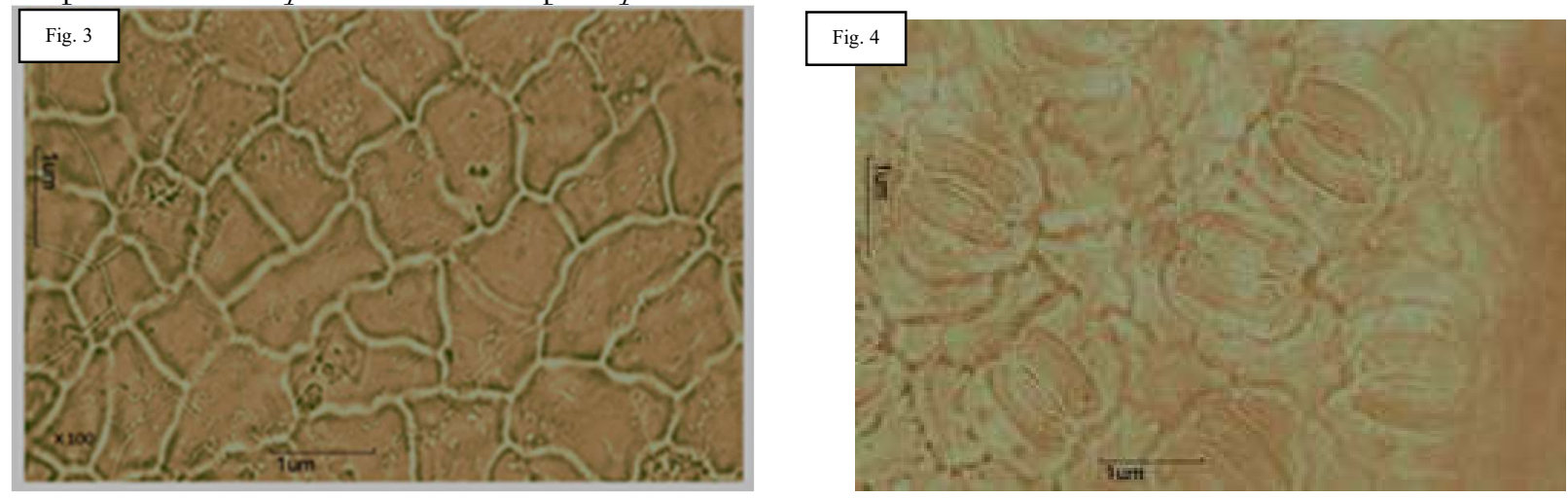

Fig. 2 : Face supérieure de la feuille du karité ; absence de stomate, cellules épidermiques polymorphes.

Fig. 3 : Face inférieure de la feuille du karité ; présence de stomate, type stomatique anomocytique périgène à subsidiaire dicyclique. 

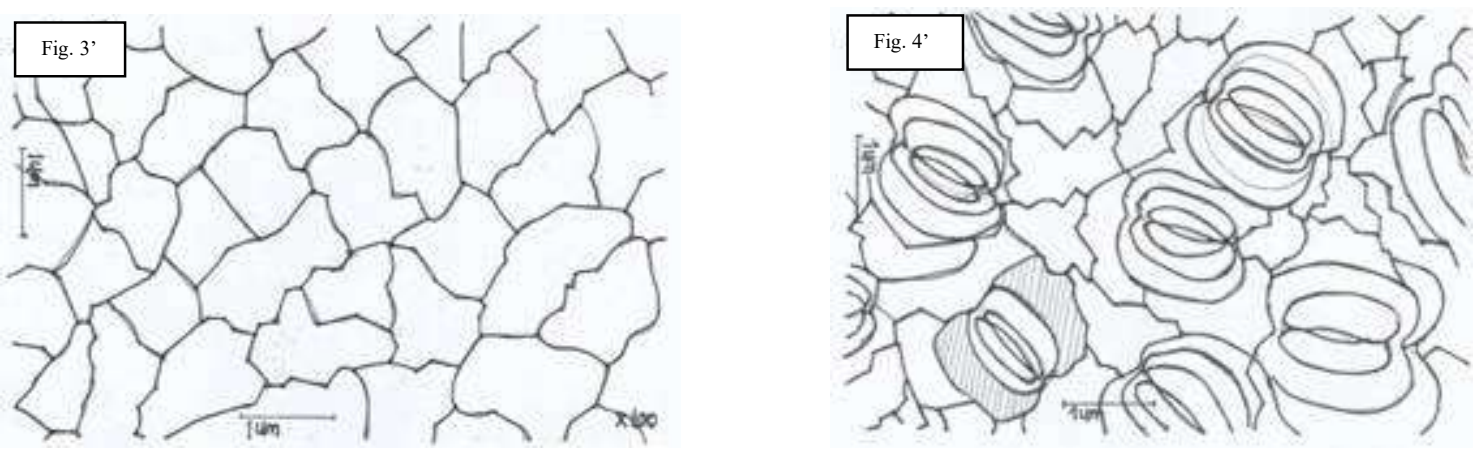

Fig. 2' : Face supérieure de la feuille du karité ; absence de stomate, cellules épidermiques polymorphes.

Fig. 3' : Face inférieure de la feuille du karité ; présence de stomate, type stomatique anomocytique périgène à subsidiaire dicyclique.

Type stomatique anomocytique périgène à subsidiaire dicyclique

\subsection{Indices stomatiques}
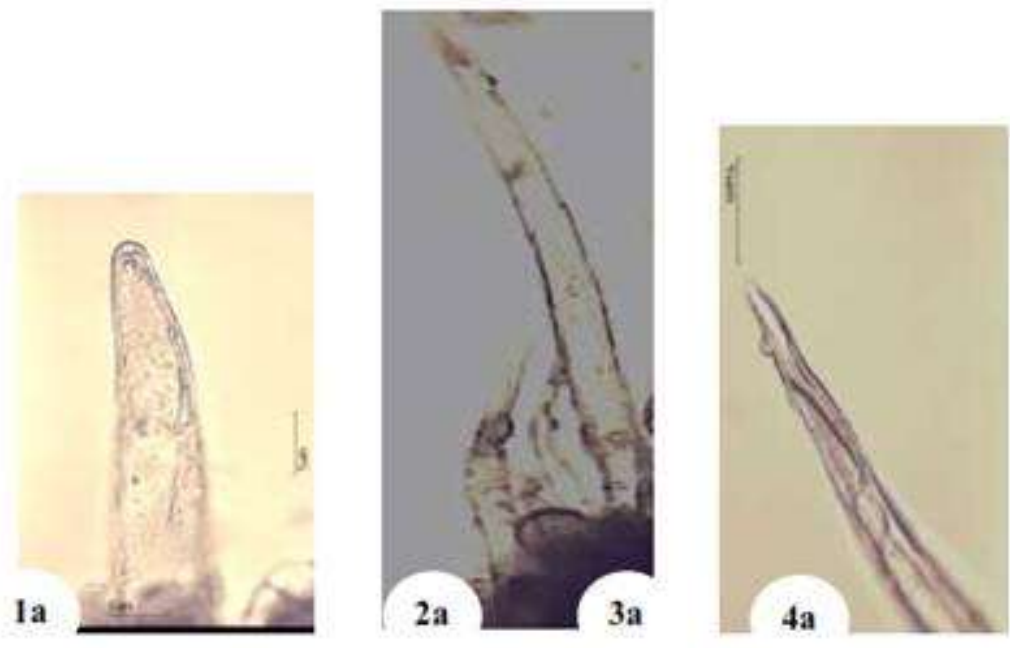

Planche II : Différents types de poils des bourgeons apicaux rencontrés chez les morphotypes de karité étudiés.

Fig. 1a et 2a : Poils simples pluricellulaires courts de 100 à $150 \mu$ dominant chez le morphotype Mbabéte ;

Fig. 3a : Poils simples pluricellulaires longs de plus de $2 \mathrm{~mm}$ dominant chez Kiankos, Meingré, Komane_Ngoïtokoro ;

Fig. 4a : Poils simples pluricellulaires moyens de 1 à 1,5 mm dominant chez le morphotype Bogrombaye. 


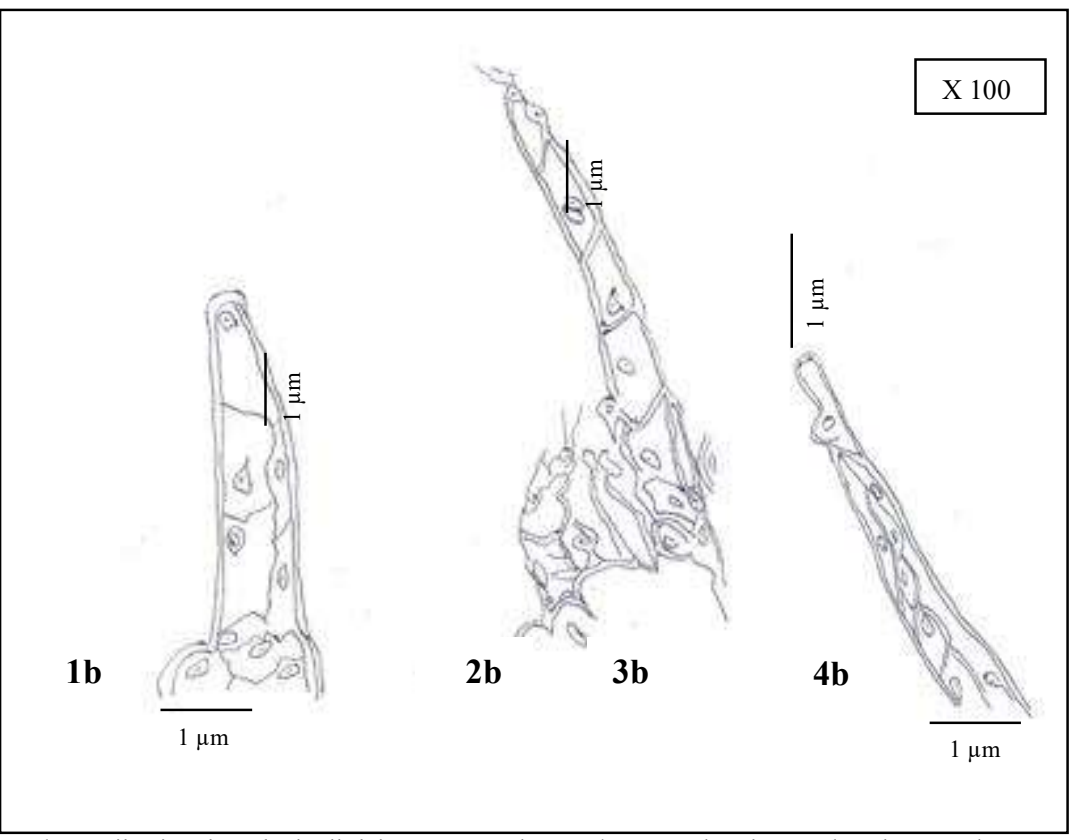

Fig. $1 \mathrm{~b}$ et 2b : Poils simples pluricellulaires courts de 100 à $150 \mu$ dominant chez le morphotype Mbabéte ;

Fig. 3b : Poils simples pluricellulaires longs de plus de $2 \mathrm{~mm}$ dominant chez Kiankos, Meingré, Komane_Ngoïtokoro ;

Fig. 4b : Poils simples pluricellulaires moyens de 1 à 1,5 mm dominant chez le morphotype Bogrombaye.

Types de poils pluricellulaires simples à taille variable

$\underline{\text { Remarque }}$ : les morphotypes de karité ont une structure hétérogène de poils. Par exemple, la photo des poils (2a et $3 \mathrm{a}$ ) qui sont ainsi de tailles différentes sont rencontrés chez le même morphotype.

4.3 Types stomatiques : Sur les échantillons que nous avons étudiés les feuilles n'ont pas de stomates sur la face supérieure. Les stomates se rencontrent chez le karité uniquement sur la face inférieure de la feuille. Bosabalidis et Kofidis (2002) ont montré que les stomates sur la surface inférieure des feuilles contribuent à un meilleur contrôle de la transpiration. Ces stomates sont accompagnés de plusieurs cellules épidermiques banales polymorphes dont la densité est faible comparativement à celles qui sont observées sur la face supérieure. Ce sont donc des feuilles hypostomatées. Chez $V$. paradoxa subsp. paradoxa, tous les cinq (5) morphotypes étudiés montrent que les stomates observés sur la face inférieure des feuilles sont de type anomocytique ou type Renonculacée périgène à subsidiaire dicyclique. En effet, les stomates sont entourés de plusieurs cellules annexes qui ressemblent aux cellules épidermiques banales. Des feuilles hypostomatées ayant un type stomatique anomocytique périgène à subsidiaire dicyclique sont considérées comme une adaptation conçue pour augmenter la conductance foliaire maximale au $\mathrm{CO}^{2}$ (Bondada et Oosterhuis, 2000). Comme la plupart des plantes des milieux extra-forestiers, les cinq (5) morphotypes étudiés ont des stomates situés à la face inférieure des feuilles, où ces stomates sont protégés des influences extérieures extrêmes (vents chauds et/ou secs, chargés d'aérosols minéraux, ensoleillés) et de leurs variations brusques et plus ou moins durables. Selon Saadoun (2005), le caractère hypostomatique des feuilles est une stratégie adaptative pour réduire les pertes en eau chez les essences végétales. La comparaison des indices stomatiques des cinq (5) morphotypes sont portés dans le Tableau 2. 
Tableau 2 : Moyenne des différents paramètres et indices stomatiques.

\begin{tabular}{|c|c|c|c|c|c|c|}
\hline $\begin{array}{l}\text { Moyennes et } \\
\text { Indices }\end{array}$ & $\begin{array}{l}\text { Morphotypes } \\
\text { Face }\end{array}$ & Bogrombaye & kiankos & Mbabète & Meingré & $\begin{array}{l}\text { Komane } \\
\text { Nooitokoro }\end{array}$ \\
\hline $\begin{array}{l}\text { Nombre } \\
\text { des cellules } \\
\text { épidermiques }\end{array}$ & $\begin{array}{l}\text { Face sup. } \\
\text { Face inf. }\end{array}$ & $\begin{array}{l}305 \\
122\end{array}$ & $\begin{array}{l}303 \\
121\end{array}$ & $\begin{array}{l}315 \\
126\end{array}$ & $\begin{array}{l}317 \\
128\end{array}$ & $\begin{array}{l}313 \\
125\end{array}$ \\
\hline $\begin{array}{l}\text { Nombres } \\
\text { de stomates }\end{array}$ & $\begin{array}{l}\text { Face sup. } \\
\text { Face inf. }\end{array}$ & $\begin{array}{c}0 \\
362\end{array}$ & $\begin{array}{c}0 \\
356\end{array}$ & $\begin{array}{c}0 \\
365\end{array}$ & $\begin{array}{c}0 \\
358\end{array}$ & $\begin{array}{c}0 \\
367\end{array}$ \\
\hline $\begin{array}{l}\text { Moyenne (a) } \\
\text { des stomates } \\
\text { observés }\end{array}$ & & 181 & 178 & 183 & 179 & 183 \\
\hline $\begin{array}{l}\text { Moyenne (b) } \\
\text { des stomates } \\
\text { observés }\end{array}$ & & 214 & 212 & 221 & 220 & 219 \\
\hline $\mathrm{n}=\mathrm{a}+\mathrm{b}$ & & 395 & 390 & 404 & 404 & 402 \\
\hline Is $=a /(a+b) * 100$ & & 45,8 & 45,6 & 45,3 & 45,1 & 45,5 \\
\hline
\end{tabular}

L'analyse du Tableau 2 montre que le nombre des cellules épidermiques banales sur la face supérieure des feuilles chez tous les morphotypes étudiés est supérieur à celui de la face inférieure. Il varie de $303 \pm 14 / \mathrm{mm}^{2}$. Les faibles valeurs sont trouvées chez Kiankos et les plus élevées sont chez Meingré. Les autres morphotypes ont des valeurs intermédiaires. Par ailleurs, le nombre de stomates des faces inférieures des feuilles des différents morphotypes étudiés varie de $356 \pm 1 / \mathrm{mm}^{2}$. Les faibles valeurs sont trouvées chez Kiankos et les plus élevées sont chez Komane_Ngoïtokoro. Les autres morphotypes ont des valeurs intermédiaires. Une densité stomatique de $303 \pm 14 / \mathrm{mm}^{2}$ obtenue dans cette étude est une caractéristique de résistance à la sécheresse. La sécheresse est la condition environnementale qui affecte le plus les caractéristiques des feuilles (XueJun et XinShi, 2000). Les plantes réagissent à la sécheresse en modifiant les processus morphologiques, physiologiques, biochimiques et métaboliques (Elfadl et Luukkanen, 2006). Ces résultats confirment les conditions du milieu savanicole où se développent les cinq (5) morphotypes étudiés.
Une densité stomatique moyenne de $308,5 / \mathrm{mm}^{2}$ obtenue en Algérie par (Belhadj et al., 2011) a été utilisée comme un indicateur dans le dépistage de la classification de huit populations sauvages de Pistaches atlantica. En outre, les résultats présentés dans le Tableau 2 montrent un indice stomatique $45,1 \pm 0,7 \%$ chez les différents morphotypes. Les indices stomatique élevés sont notés chez le morphotype Bogrombaye ( $I s=45,8 \%$ ) et les plus faibles chez le morphotype Meingré (Is = 45,1\%). Les morphotypes Kiankos, Mbabéte et Komane_Ngoïtokoro présentent des indices intermédiaires. Cependant, les indices des stomates à eux seuls n'ont pas révélé une réponse à la phénologie liée à la précocité ou tardivité de la maturation agronomique respectivement chez les morphotypes Bogrombaye et Meingré signalée en ethnobtanique par Djekota (2008), suggérant que cette phénologie ne serait pas uniquement liée à l'indice stomatique. La comparaison de ces indices stomatiques par l'écart réduit des proportions a donné les résultats qui sont présentés dans le Tableau 3. 
Tableau 3 : Ecart réduit des indices stomatiques des morphotypes pris 2 à 2 .

\begin{tabular}{l|c|c|c|c|c}
\hline MORPHOTYPES & Bogrombaye & kiankos & Mbabète & Meingré & $\begin{array}{c}\text { Komane_ } \\
\text { Ngoïtokoro }\end{array}$ \\
\hline Bogrombaye & 1,5 & - & & & \\
kiankos & 1 & 2,5 & - & & \\
Mbabète & 0,5 & 2 & 0,5 & - & - \\
Komane_Ngoïtokoro & 1 & 0,5 & 2 & 1,5 & - \\
\hline
\end{tabular}

Des valeurs lues sur la table $t$ au seuil de signification $\alpha=0.05$ montre sur Tableau 3 que les taxa les plus proches sont Bogrombaye et Meingré, Komane_Ngö̈tokoro et Kiankos mais aussi Meingré et Mbabéte sont proches même si leur distance est relativement plus grande que dans le premier cas. Les taxa les plus éloignés du point de vue indice stomatique sont Mbabéte et Kiankos. Alors, chez tous les cinq (5) morphotypes l'hypothèse d'égalité des 2 proportions est acceptée : c'est-à-dire qu'il n'y a pas une différence significative entre les morphotypes au point de vue indice stomatique. Ces résultats confirment le rôle important que les stomates jouent dans la transpiration des végétaux.

4.4 Proposition d'une clé partielle avec des caractères micro morphologiques : Les principaux caractères micro morphologiques étudiés sont résumés sur le Tableau 4.

Tableau 4 : Caractères micro morphologiques étudiés.

\begin{tabular}{|c|c|c|c|c|c|c|}
\hline \multicolumn{2}{|c|}{ MORPHOTYPES } & Bogrombaye & Kiankos & Mbabète & Meingré & $\begin{array}{c}\text { Komane } \\
\text { Ngoütokoro }\end{array}$ \\
\hline \multirow{3}{*}{$\begin{array}{l}\text { Type de } \\
\text { Poils }\end{array}$} & $\begin{array}{l}\text { Poils simple } \\
\text { Court }<150 \mu\end{array}$ & - & - & + & - & - \\
\hline & $\begin{array}{l}\text { Poils moyen de } \\
1 \text { à } 1,5 \mathrm{~mm}\end{array}$ & - & - & - & + & - \\
\hline & $\begin{array}{l}\text { Poils long de }> \\
1,5 \mathrm{~mm}\end{array}$ & + & + & - & - & + \\
\hline $\begin{array}{c}\text { Type } \\
\text { stomatique }\end{array}$ & $\begin{array}{l}\text { Anomocytique } \\
\text { Périgène à } \\
\text { subsidiaire } \\
\text { dicyclique }\end{array}$ & + & + & + & + & + \\
\hline \multirow{2}{*}{$\begin{array}{c}\text { Indice } \\
\text { stomatique }\end{array}$} & $\begin{array}{l}\text { Indice } \\
\text { stomatique } \\
\leq 45,5 \%\end{array}$ & - & - & + & - & - \\
\hline & $\begin{array}{l}\text { Indice } \\
\text { stomatique } \\
\geq 45,5 \%\end{array}$ & + & + & - & + & + \\
\hline
\end{tabular}

4.5 Clé de détermination des morphotypes du karité à l'aide des caractères micro morphologiques: Sur la base des caractères liés aux différents types de poils des bougeons apicaux (Tableau 4), la clé de détermination ci-après est proposée pour améliorer la connaissance et l'identification des morphotypes étudiés. 
1. Poils simples courts de $<150 \mu$...Mbabéte

1'. Poils simples longs $>150 \mu$

2. Poils simples moyens de 1 à $1,5 \mathrm{~mm}$

\section{...Meingré}

2'. Poils simples longs de plus de $2 \mathrm{~mm}$

3. Indice stomatique $<45,5 \%$

\section{Komane_Ngoïtokoro}

3'. Indice stomatique $>45,5 \% \ldots$ Bogrombaye, Kiankos.

De cette clé partielle, la taille des poils dominants ainsi que l'indice stomatique apparaissent comme des caractères importants qui permettent de distinguer de façon précise 4 groupes de morphotypes :

\section{$4 \quad$ CONCLUSION}

Les cinq (05) morphotypes de karité qui ont fait l'objet de recherche des caractères micro morphologiques discriminants susceptibles d'améliorer leur identification taxonomique montrent l'absence de stomates sur la face supérieure des feuilles. Les stomates observés uniquement sur la face inférieure des feuilles sont de type anomocytique périgène à subsidiaire dicyclique. La densité stomatique est $362 \pm 5 \mathrm{st} / \mathrm{mm}^{2}$ et un indice stomatique qui varie de $45,1 \pm 0,7 \%$ chez les morphotypes de karité étudiés confirment leur adaptation aux

\section{REFERENCES}

Arbonnier M., 2000. Arbres, arbustes et lianes des zones sèches d'Afrique de l'Ouest. Cirad.

Barfod A., 1988. Leaf anatomy and its taxonomic significance in phytelephantoïd palms (Araceae). Nord. J. Bot. 8: 341-348.

Belhadj S., Derridj A., Aigouy T., Gers C., Gauquelin T., Mevy J.P., 2007a. Comparative morphology of leaf epidermis in eight populations of Atlas pistachio (Pistacia atlantica Desf., Anacardiaceae). Microsc. Res. Tech. 70: 837-846.

Safia Belhadj, Arezki Derridj, Alfonso Moriana, Maria Del Carmen Gijon, Jean-Phillipe Mevy and Thierry Gauquelin, 2011. Comparative analysis of stomatal
Poils simples courts dominants (Mbabéte); Poils simples moyens dominants (Meingrê) ; Poils simples longs dominants avec indice stomatique faible (Komane_Ngoïtokoro) ;

Poils simples longs dominants avec indice stomatique élevé (Bogrombaye, Kiankos).

Cette clé montre l'importance de l'apport des caractères micro morphologiques liés aux poils apicaux dans la détermination des différents morphotypes. Ce travail montre que les caractères des poils apicaux, l'indice stomatique et le type stomatique sont de bons descripteurs des différents morphotypes chez Vitellaria paradoxa subsp. paradoxa.

milieux secs. Les résultats de ce travail montrent également que les poils observés sont pluricellulaires et peuvent être simples courts, moyens et longs avec une nette dominance des poils courts. En perspective, la combinaison des caractères macro et micro morphologiques permettra probablement de préciser les caractères ou associations de caractères qui définissent le mieux un morphotype ou un groupe de morphotype chez Vitellaria paradoxa subsp. Paradoxa.

characters in eight wild atlas pistachio populations (Pistacia atlantica Desf.; Anacardiaceae). International Research Journal of Plant Science (ISSN: 21415447) Vol. 2(3) pp. 060-069.

Bondada B.R., Oosterhuis D.M., 2000. Comparative epidermal ultrastructure of Cotton (Gossypium hirsutum L.) leaf, bract and capsule wall. Ann. Bot. 86:11431152.

Bosabalidis A.M., Kofidis G., 2002. Comparative effects of drought stress on leaf anatomy of two olive cultivars. Plant Sci. 163:375-379.

Byakagaba Partick, Gerald Eilu, John Bosco L. Okullo, Susan B. Tumwebaze and Edward N. Mwavu, 2011. Population 
structure and regeneration status of Vitellaria paradoxa (C.F/Gaertn) under dif regions in Uganda. Agricultural journal 6 (1): 14-22, ISSN: 1816-9155.

Couderc H., 1979. Etudes des stades de jeunesse chez plusieurs espèces du genre Anthyllis. Bull. soc. Bot. Fr., 126. $\mathrm{N}^{\circ} 3: \mathrm{p}$ 93-98.

Decamps O. et Saadoun N., 1993. L’ontogénie stomatique en Biosystématique. Gaussenia, $8: 38-42$.

Diop D., 2008. Contribution à l'étude biosystématique des espèces du genre Ficus (Moraceae) au Sénégal. Thèse de doctorat de 3ème cycle. Faculté des sciences et techniques, UCAD. Dakar.

Djekota C. N., 2008. Caractérisation macromorphologique du Butyrospermum parkii (Vitellaria paradoxa C.F Gaertn, subsp. paradoxa) dans la région de Mandoul au Tchad. Mémoire de D.E.A ; Université Cheikh Anta Diop, Dakar/Sénégal.

Djekota C. N., Diouf D., Sané S., Mbaye M.S. and Noba K., 2014a. Morphological characterization of shea tree (Vitellaria paradoxa C.F.Gaertn subsp paradoxa) populations in the region of Mandoul in Chad. Academic Journal, International Journal of Biodiversity and Conservation. Vol. 6 (2), pp. 184-193. ISSN

$2141-$

243X.http://www.academicjournals.org $\angle \mathrm{IJBC}$.

Djekota C. N., 2014b. Etude de la variabilité morphologique et taxonomie des Variétés chez le Karité (Vitellaria paradoxa C.F. Gaertn sous-espèce paradoxa, Sapotaceae) dans la région du Mandoul au Tchad. Thèse de Doctorat Unique. Faculté des Sciences et Techniques, UCAD. Dakar/Sénégal.

Djekota C.N., Mouga M., Djimramadji A., Djelassem B., Mbayngone E., Maïga R. D., Rimgoto K., Noubady D., 2014c. Potentiel karité au Tchad (Vitellaria paradoxa C.F. Gaertn. subsp. paradoxa).
Journal of Animal \& Plant Sciences, 2014. Vol. 23, Issue 3: 3646-3656. http://www.m.elewa.org/JAPS; ISSN 2071-7024.

Elfadl MA, Luukkanen O., 2006. Field studies on the ecological strategies of Prosopis juliflora in dry land ecosystem 1. A leaf gas exchange approach. J. Arid Env. 66:1-15.

Guyot, M. R., 1966. Les stomates des Ombelles. Bull. Soc. Bot. Fr., 113 : 244272.

Gwali Samson, Grace Nakabonge, John Bosco Lamoris Okullo, Gerald Eilu, Philip Nyeko, Peter Vuzi, 2012. Morphological variation among shea tree (Vitellaria paradoxa subsp. nilotica) 'ethnovarieties' in Uganda. Genet Resources Crop Evol 59:1883-1898 : $\quad 10.1007 / \mathrm{s} 10722-012$ 9905-8.

Lamien N., Tigabu M., Guinko S., Oden P.C., 2007. Variations in dendrometric and fruiting characters of Vitellaria paradoxa populations and multivariate models for estimation of fruit yield. Agrofor Syst 69:1-11. doi:10.1007/s10457- 0069013-x

Mbaiguinam M., Mbayhoudel K. \& Djekota C. N., 2007. Physical and Chemical Characteristics of Fruits, Pulps, Kernels and Butter of Shea Butyrospermum parkii (Sapotaceae) from Mandoul, Southern Chad. Asain Journal of Biochemistry 2 (2): 101-110. ISSN 1815-9923.

Mbaye M.S., Noba K., Sarr R.S., Kane A., Sambou J. M. \& Ba A.T., 2001. Eléments de précision sur la systématique d'espèces adventices du genre Corchorus L. (Tiliaceae) au Sénégal. African Journal of Science and Technology 2(1): 51-61.

Metcalfe C.R. \& Chalk L., 1950 - Anatomy of the Dicotyledons.

Mohagir A. M., Kamga R., Kapseu C. \& Abi C. F., 2009. Optimization of some pretreatment involved in the press extraction of Shea (Vitellaria paradoxa 
Gaertner F.) Butter. Asian Journal of Applied Sciences, 2 (4): 372-384, ISSN 1996-3343.

Nafan D., N'guessan A., Koffi E. \& Sangaré A., 2007. Evaluation des performances de quelques descripteurs quantitatifs et leur utilisation dans la structuration de la population d'un parc naturel de karité en Côte d'Ivoire. Plants Genetic Resources Newsletter 152 : 65-72.

Noba K., 1990. Contribution à l'étude biosystématique de 3 espèces du genre Boerhaavia L.: B. diffusa L., B. erecta L., B. repens L. Thèse de Doctorat de 3éme cycle, UCAD, Dakar.

Nyarko G., Mahunu G. K., Chimsah F. A., Yidana J. A., Abubakari A-H., Abagale F.K., Quainoo A. \& Poudyal M., 2012. Leaf and fruit characteristics of Shea (Vitellaria paradoxa) in Northern Ghana. Research in Plant Biology, 2(3): 38-45, 2012 ISSN: 2231-5101

INSEED, 2012. Résultats définitifs par Souspréfecture du Deuxième Recensement Général de la Population et de l'Habitat (RGPH2)/Institut National de la Statistique des Etudes Economiques et Démographiques ; $121 \mathrm{p}$.

Saadoun N. \& Decamps O., 1991. A new stomatal type in Chenopodiaceae. Plant Syst. Evol. 177 :11-16.

Saadoun N., 2005. Réponse adaptative de l'anatomie des Chénopodiacées du Sahara algérien à des conditions de vie d'aridité extrême. Sécheresse 16(2): 121124.

Sanou H.P.N., Lovett \& Bouvet J. M., 2006. Comparison of quantitative and molecular variation in agroforestery populations of the shea tree (Vitellaria paradoxa C.F. Gaertn) in Mali. Molecular Ecology 14: 2601-2610.

SIDRAT, 2013a. Atlas du Tchad/Projet «Programme d'Information pour le Développement Durable et l'Aménagement du territoire»; République du Tchad.
Soro D., N’Da-Adopo A., Da K.P., Traoré D., 2004a. Lutte contre les parasites chez le karité : Agron. Afr., 16(3): 21-28.

Soro D., Ouattara D., Da K..P., Traoré D., 2004b. Efficacité de l'émondage contre Loranthaceae ou guis du karité : cas du parc naturel à karités de Tengrela, dans le Nord de la Côte d'Ivoire : Ann. de Bot. Afr. de l'Ouest, (3): 87-95.

Turquet J., 1910. Recherches anatomiques sur les Combretum africains. Thèse série A, Paris, $645 \mathrm{p}$.

Ugese F.D., Baiyeri P.K., Mbahbn, 2010. Agroecological variation in the fruits and nuts of shea butter tree (Vitellaria paradoxa C. F. Gaertn.) in Nigeria. Agrofor Syst 79(2):201-211.

XueJun D., XinShi Z., 2000. Special stomatal distribution in Sabina vulgaris in relation to its survival in a desert environment. Trees. 14: 369-375. 